\title{
A Laser Speckle Method for Measuring Displacement Field. Application to Resistance Heating Tensile Test on Steel
}

\author{
C. Pradille ${ }^{a}$, M. Bellet ${ }^{b}$ and Y. Chastel ${ }^{c}$ \\ MINES-ParisTech, CEMEF, UMR CNRS 7635, Sophia Antipolis, France \\ achristophe.pradille@mines-paristech.fr, ${ }^{b}$ michel.bellet@mines-paristech.fr, \\ cyvan.chastel@mines-paristech.fr
}

Key words: full field measurement, laser speckle, cross-spectrum, cross-correlation

\begin{abstract}
Strain field measurement with non intrusive techniques is needed in order to characterize the behaviour of steels at high temperatures subjected to small displacements. In this work we present a technique based on laser-produced speckles coupled with a cross-correlation crossspectrum method. This method proves more accurate than cross-correlation for small displacements. The laser wave length used $(532 \mathrm{~nm})$ allows to perform strain measurements, even with heat radiation.
\end{abstract}

\section{Introduction}

Understanding of solidification crack phenomena is a challenge. On one hand, industrial concerns are considerable in terms of productivity and product quality. On the other hand, physical mechanisms of hot tearing are far from being well understood, which makes it a scientific challenge. For this highly multiphysics problem, one of the main difficulties lies in the rheological characterization of metallic alloys, at high temperature and in the semi-solid state. Actually, there are only few data available for constitutive laws of industrial steel for solidification conditions (ie high temperature and very low strain and strain rate). Rheological characterization under tensile conditions has been carried out using a direct resistance heating machine named Taboo. The Taboo set-up allows cyclic loadings at very low strain rate $\left(10^{-3}-10^{-4} \mathrm{~s}^{-1}\right)$ between $900^{\circ} \mathrm{C}$ and the solidus temperature. A problem with resistance heating techniques is the significant thermal gradient along the sample, which causes heterogeneous strains. Due to the spatial heterogeneity of the strain field, the displacement field needs to be known on the entire sample. The speckle correlation photography has several advantages like full field and non destructive measurement. However, this method is classically limited in temperature (around $900^{\circ} \mathrm{C}$ ). The reason for this is that the speckle is usually produced with painting [1]. Furthermore, at high temperatures these methods based on optical measurements are also very sensitive to the visible spectrum, in particular in the red wave length. All these limitations lead us to develop a new method based on laser-produced speckles. Since, as for steel, radiation is absent in the green wave length around $1500^{\circ} \mathrm{C}$, a green laser is used. In this work we also present a technique of image analysis based on a cross-spectrum method. This method proves more accurate than cross-correlation technique for small displacement and more efficient for quickly analysing two pictures within only a few milliseconds. In this paper, we first describe the experimental set-up; then we present the measurement technique. Thanks to this method we obtain full field measurements.

\section{Experimental Set-up}

Taboo Tensile Test Machine. The "Taboo" machine (Fig. 1) is a thermo-mechanical simulator which has been developed at Cemef to investigate the behaviour of steels at very high temperature up to the solidus temperature and possibly in the semi-solid state. Before applying the loading cycle, the sample is directly heated by Joule effect using a continuous current. The temperature monitoring of the sample is achieved as follows. A thermocouple is welded in the middle of the specimen. During the heating and loading phases of the test, the current intensity is dynamically controlled in order to prescribe a desired temperature. An alternative monitoring procedure consists 
in using a thermal camera to measure the temperature field on the specimen surface. During the heating stage, the mobile grip displacement is monitored so that no external force is applied on the specimen. Once the desired temperature is reached, the mechanical test starts and the tensile force is measured by a load cell. The measurement of force vs displacement curves requires a high temperature extensometry technique. For that purpose, a non-contact method has been developed and is presented in the next section.
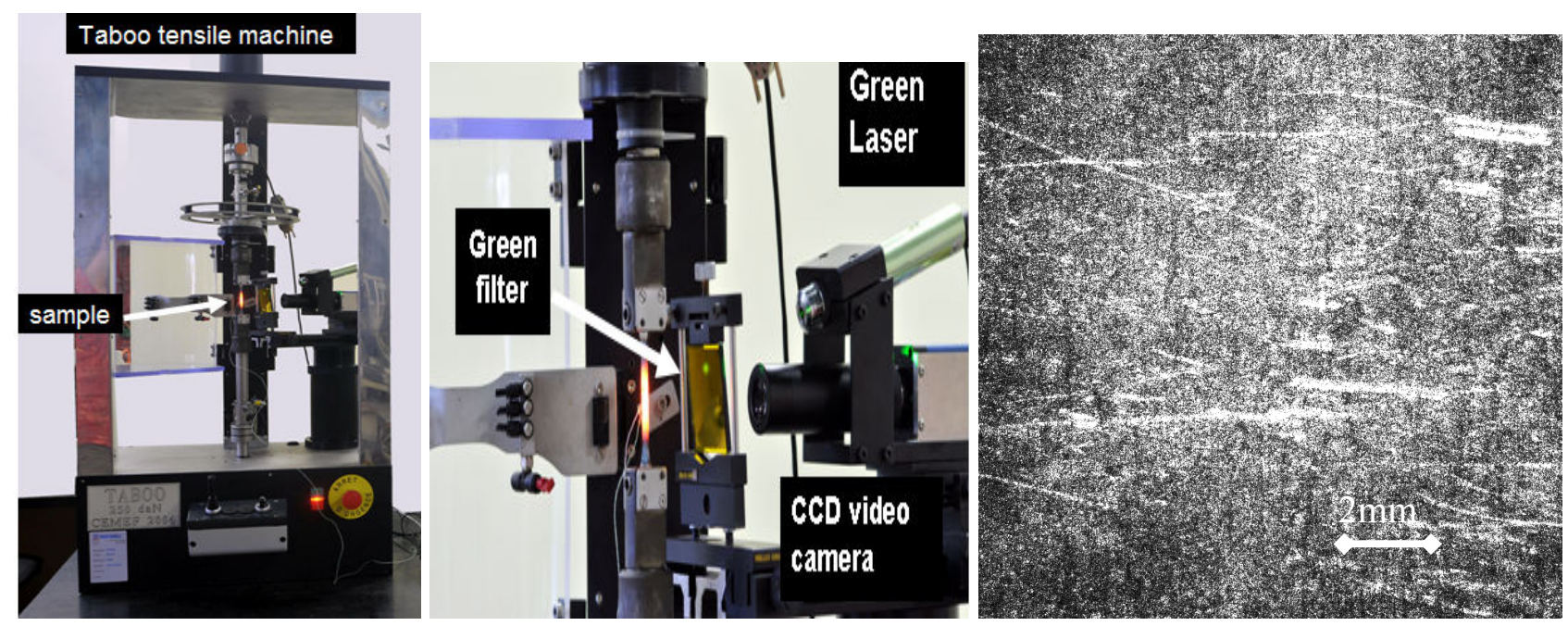

Fig. 1: "Taboo" tensile machine (left), laser speckle equipment (centre) and a typical laser speckle pattern (right).

Speckles Set-up. A laser is projected on the area in which the displacement field needs to be measured. The laser beam is enlarged by a microscope lens that allows illuminating a large area on the central part of the tensile sample (typically $10 \times 6 \mathrm{~mm}$, the specimen being $90 \mathrm{~mm}$ long and 6 $\mathrm{mm}$ wide). Speckle images arising from local interference phenomena due to surface roughness are captured by a CCD camera and are simultaneously transferred to a computer. The size of analysed pattern areas is $1920 \times 1080$ (about 2 Mpixels). Each pixel can store a grey scale 8-bit value ranging from 0 to 255. During the experiment, a series of patterns can be either collected for post treatment later on or correlated and analysed for the control loop. The correlation between initial and current speckle patterns provides the displacement, as explained in the next section.

\section{Image Correlation Techniques}

The surface is considered to change relatively slowly during the experiment. Consequently, over a small time step, strains are low and the observed images are simply translated. In the following paragraph a technique of image analysis based on a coupled cross-correlation / cross-spectrum technique is presented. This technique is used to obtain the displacement field from two images. This coupled method allows characterizing the displacement within a few milliseconds with a subpixel resolution for a large range of displacement (from less than 1 pixel to 20 or more pixels).

The cross-spectrum and the cross-correlation methods have different advantages. Cross-correlation method is less accurate for small displacements and less rapid than cross-spectrum method but it is more accurate for large displacement.

The Cross-spectrum Method [2]. Consider a plane surface covered by a speckle pattern where the image intensity at time $t_{0}$ is defined by $f(x, y)$. The Fourier transform $F(u, v)$ is defined as:

$$
T F\{f(x, y)\}=F(u, v)=\iint f(x, y) e^{-2 i \pi(u x+v y)} d x d y .
$$

Consider now a second image where the intensity at time $t_{1}=t_{0}+\Delta t$ is defined as $g(x, y)$. If function $g(x, y)$ is written as a translation of $f(x, y): g(x, y)=f\left(x-x_{0}, y-y_{0}\right)$, and defining the 
cross-spectrum $I(u, v)$ as the multiplication of $F(u, v)$ by the complex conjugate of $G(u, v)\left(\right.$ denoted $\left.G^{*}(u, v)\right)$, we get:

$$
I(u, v)=F(u, v) G^{*}(u, v)=F(u, v) F^{*}(u, v) e^{2 i \pi\left(u x_{0}+v y_{0}\right)}=|F(u, v)|^{2} e^{2 i \pi\left(u x_{0}+v y_{0}\right)} .
$$

Eq. 2 shows that the displacement can be expressed according to two components corresponding to two different directions. The phase can consequently be decomposed into two expressions. The displacement is obtained from a linear fit of the expression of the phase (Fig. 2a). It seems quite simple to obtain a measure of the displacement between two different pictures with a crossspectrum method. But, at high spatial frequencies, the noise and/or a bad sampling can lead to a zero value of the phase instead of showing the expected random variation (between $-\pi$ and $+\pi$ ). Moreover, fitting the phase slopes is not precise when the displacement is larger than 1 pixel. In fact, in this case the phase varies between $-\pi$ and $+\pi$. A displacement can not be accurately determined when a poor sampling is added to a slope of the phase, as illustrated in Fig. $2 \mathrm{~b}$.

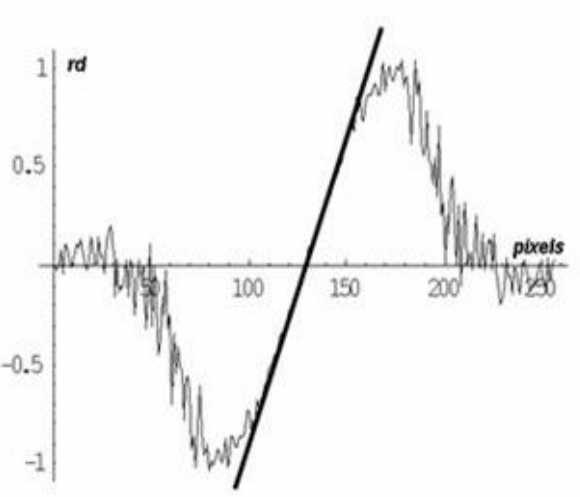

a)

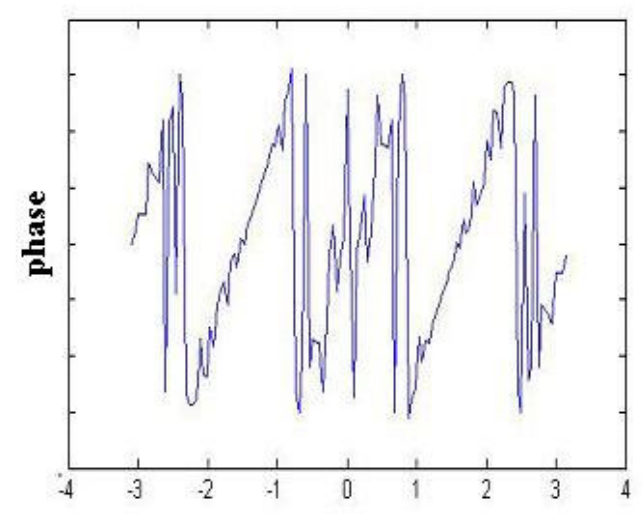

b)

Fig. 2: a) Example of displacement calculations. The phase falls down to 0 for high spatial frequencies; b) Example of the phase for a displacement larger than 1 pixel.

The Cross-correlation Method. In most papers dealing with field measurement (e.g. [3, 4]), the cross-correlation technique is reported to be used. In practice, the Wienner-Kinchine theorem states that the cross-correlation can be written as the inverse Fourier transform of Eq. 2 and these two functions contain equivalent information. However, it is more convenient to use the cross-spectrum method to measure very small displacements (smaller than pixel). Defining the Fourier transform of $I(u, v)$ as $I(\xi, \eta)$, we write:

$$
I(\xi, \eta)=\iint I(u, v) e^{2 i \pi(\xi u+\eta v)} d u d v=\iint|F(u, v)|^{2} e^{2 i \pi\left(u\left(\xi-x_{0}\right)+v\left(\eta-y_{0}\right)\right)}=\bar{I}\left(\xi-x_{0}, \eta-y_{0}\right) .
$$

A Coupled Cross-correlation - Cross-spectrum Technique. As mentioned above and shown in Fig. 2, for a large displacement (over 10 pixels), the use of the cross-spectrum method becomes inappropriate. Moreover, a shear mode might be difficult to analyse. Indeed, a minor displacement in the non-longitudinal direction creates a noise for the phase in the principal direction. In this case, it is suggested to use a coupled cross-correlation - cross-spectrum technique (CC-CS). The principle of the method is presented in Fig. 3. As shown is this figure, a cross-correlation is first used to determine a displacement of the order of one pixel. One of the two images is shifted about one pixel and a cross-spectrum analysis is then used to determine the sub-pixel scale displacement. 
Finally, displacement between two images is calculated by summing the displacements obtained by the two methods. In the technique presented here, a single image is divided into a grid of sub-pictures. Their size varies between $8 \times 8$ and $256 \times 256$ pixels. The algorithm is then applied to each couple of sub-pictures to determine the local displacement field between them. Therefore, the size and number of sub-pictures give the maximum spatial resolution of the displacement field. Sub-elements can be perfectly placed side-by-side or overlapped.

In the next sections, a numerical validation and an application of real tensile tests are presented, to demonstrate the reliability of the proposed method.

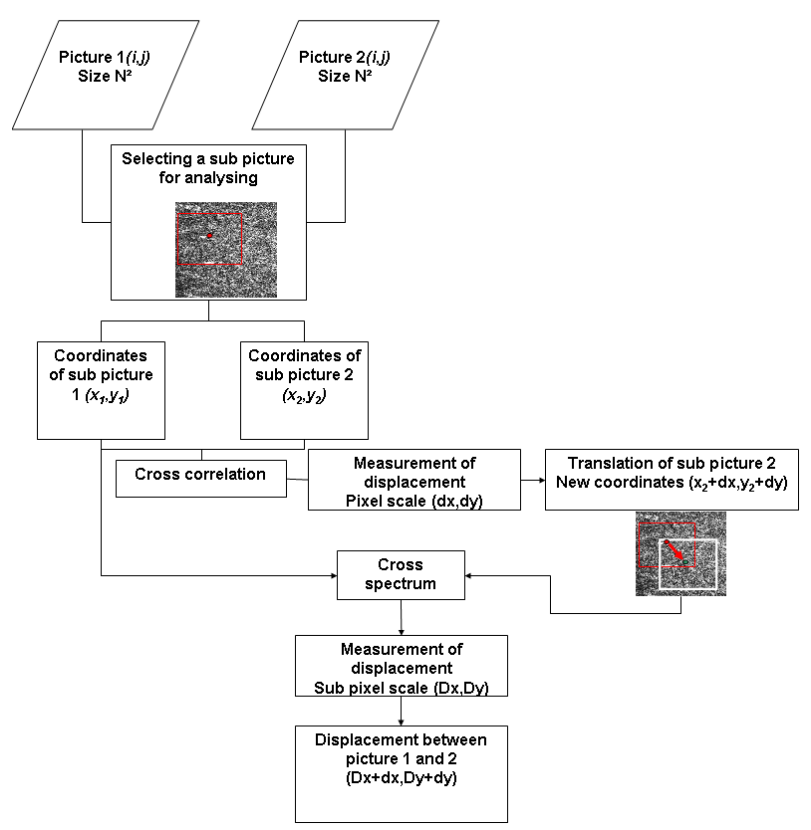

Fig. 3: Schematic algorithm of the coupled crosscorrelation cross-spectrum (CC-CS) technique.

\section{Numerical Validation}

Two tests are defined hereafter. They consist in applying a virtually prescribed displacement field to the speckle image ( $800 \times 800$ pixels) as shown in Fig. 1, using the software GIMP.

Numerical Rigid Body Rotation. The image is numerically rotated by $2^{\circ}$ around the image centre. Fig. 4a shows the displacement vectors which were calculated with the hybrid CC-CS algorithm shown in Fig. 3. The displacement is small in the centre of specimen and increases linearly towards the border of the analysed box, as expected.

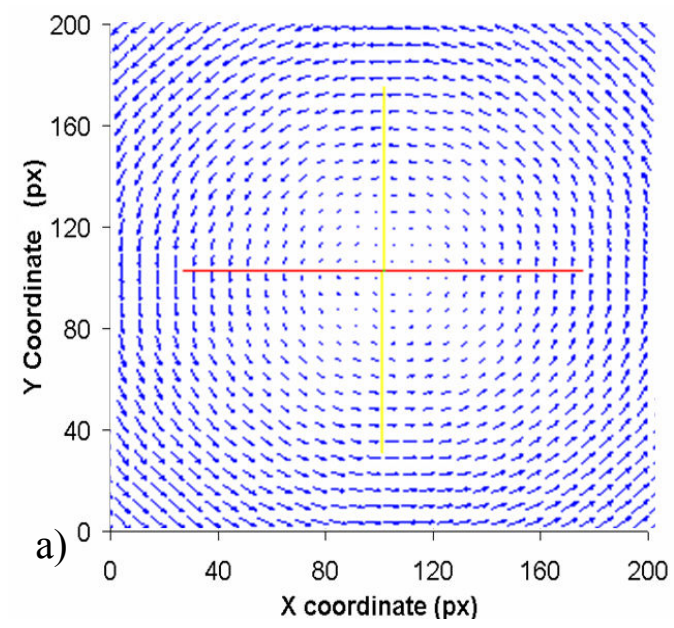

b)

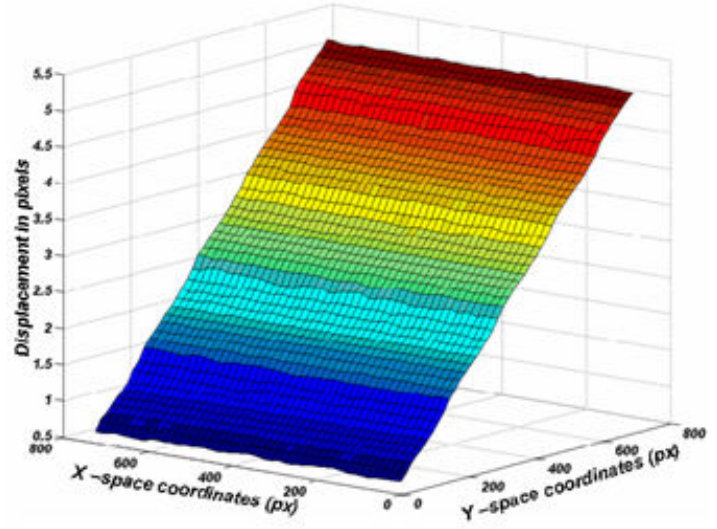

Fig. 4: Numerical application of the CC-CS technique: a) Displacement vectors for a prescribed rigid body rotation (central region only), b) Horizontal displacement field after tensile test.

Numerical Tensile Test. The same initial picture is elongated along one direction, by 5 pixels. The displacement after coupled CC-CS analysis is presented in Fig. 4b. A linear variation of the displacement field from the left side to the right side boundary can be observed. It can be noted that 
the displacement at the fixed boundary is found to be very close to zero and that the displacement field is continuous throughout the image. This demonstrates the sub-pixel accuracy of the proposed hybrid method.

\section{Application to a Real Tensile Test}

A notched thin sample ( $25 \mathrm{~mm}$ wide, $120 \mathrm{~mm}$ long, $1 \mathrm{~mm}$ thick) is subjected to a tensile load. One side of the specimen is illuminated by the laser, while the other side is covered by a speckle-like pattern created using black and white spray paints. All images are recorded on both sides of the sample using two cameras. Fig. 6a presents both speckle patterns. Different comparisons are applied on captured images, first for different correlation algorithms, and second for different types of speckles.

Comparison of Results Obtained on the Painted Surface. The presented algorithm and a commercial algorithm are both used to determine displacement fields on the painted surface of the sample. Fig. 5a and b show a comparison of the fields determined by two different algorithms, and Fig. 5c presents more precisely a displacement curve along the $\mathrm{Y}$ transverse axis. In both cases the results are similar.

a)

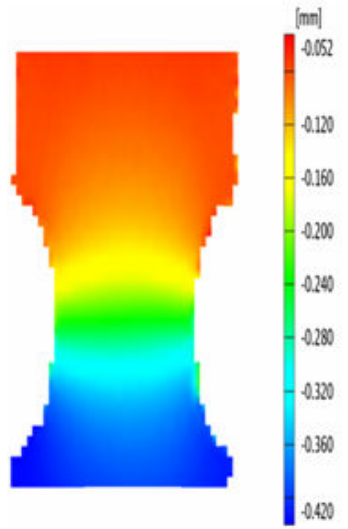

b)

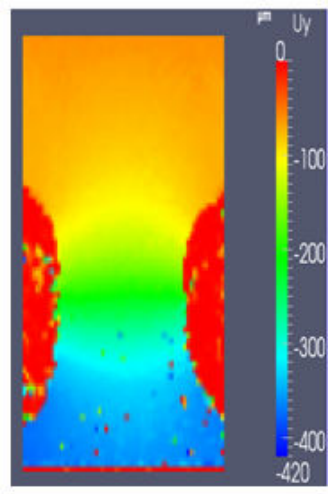

c)

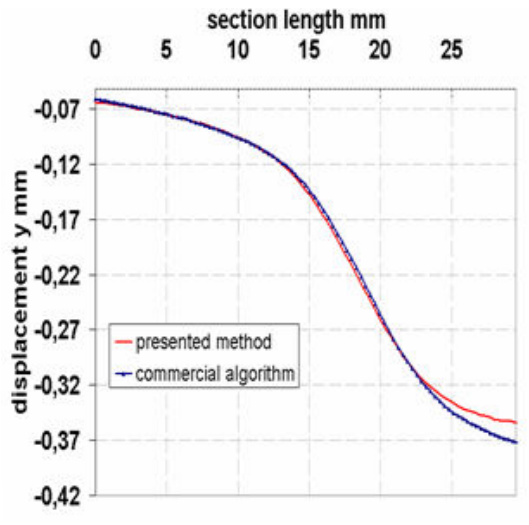

Fig. 5: Distribution of vertical displacement component, as determined by ARAMIS* (a) and the hybrid algorithm (b) on the painted face. On the right (c), comparison between displacement curves.

Comparison of Displacement Obtained with the Two Methods. The analysed area is not the same on the two faces of the sample; only a quarter of the sample is analysed. Fig $6 \mathrm{~b}$ shows the comparison of displacement curves obtained with the laser speckle method and the painted speckle.
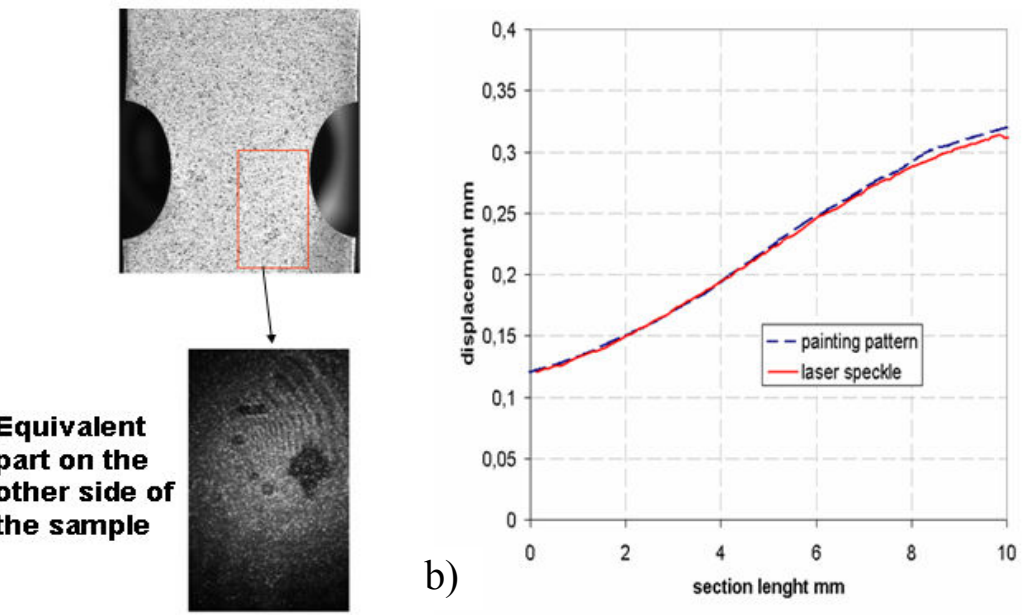

Fig. 6: a) Black and white painting on the sample surface, and the equivalent area on the other face over which the laser is projected. b) Comparison of displacements obtained with the two techniques on the two faces of the sample. 


\section{Application to Hot Tensile Test}

Following previous validations, the hybrid CC-CS technique is now applied to tests performed at high temperature $\left(1200^{\circ} \mathrm{C}\right)$. Fig 7 shows the analysis of a laser speckle pattern in order to determine a displacement field. As shown in Fig 7b, accurate correlation is possible except in areas exposed to severe oxidation (red circle area). As expected, the method remains reliable for high temperature except in the region where the surface aspect changes notably between two shots. Therefore, a careful protection against oxidation like argon gas along the surface is necessary. Another concern at very high temperature remains thermal gradients in gas boundary layers, or thermal plume detachment that could affect the accuracy of the speckle method. Using argon protection may help avoid such problem, but this needs to be confirmed.

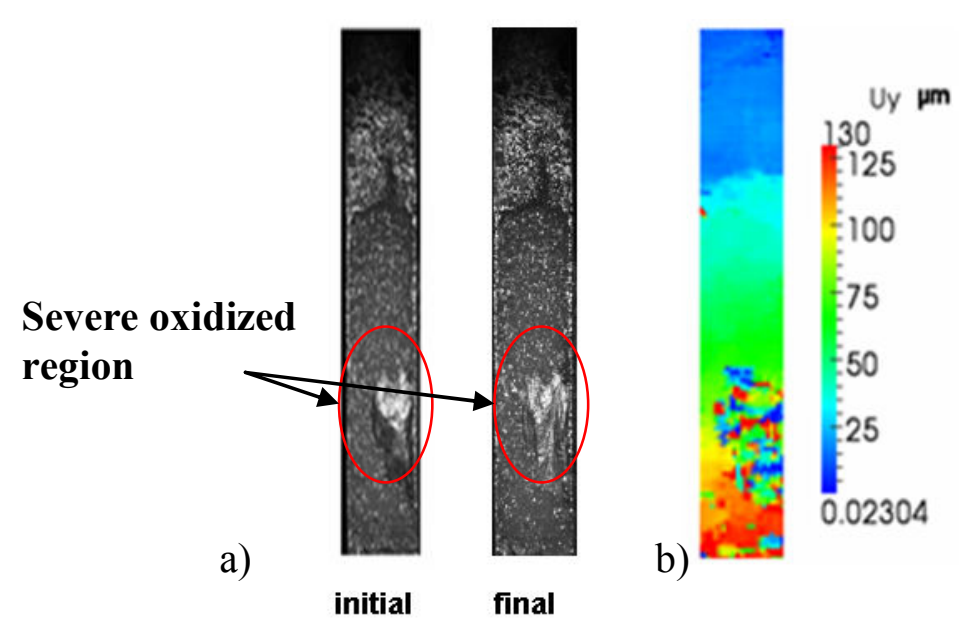

Fig. 7: a) Example of speckle pattern on a hot sample $\left(1200^{\circ} \mathrm{C}\right)$. b) Measured vertical displacement field.

\section{Summary}

This paper presents a non-contact method using laser speckles to evaluate the displacement field during a mechanical test. As shown, the method was validated on virtual and real tensile tests. Moreover an original algorithm based on a coupled correlation cross-spectrum method proves efficient for sub-pixel scale measurement and faster than algorithms commonly used. Given the calculation times, dynamic control is possible with such a speckle method. A first approach also shows the possibility to apply this method at high temperature. Besides, a finite element method is currently being developed to simulate a combined electrical thermal and mechanical test. And an inverse analysis procedure will be developed to determine high temperature behaviour.

\section{Acknowledgement}

This study has been conducted in the framework of the cooperative project "Cracracks" supported by the French Agence Nationale de la Recherche (ANR).

* ARAMIS is a registered trademark of GOM GmbH (http://service.gom.com)

\section{References}

[1] R. Knockaert, PhD thesis, MINES-ParisTech, (2001)

[2] C. Grec, C. Aime, M. Faurobert, G. Ricort, F. Paletou, A\&A, Vol. 463, p. 1125-1136, (2006)

[3] L. Chevalier, S. Calloch, F. Hild, Y. Marco, Eur J mech A/solids, Vol. 20, p.169-187,

[4] H.J. Huan, A. Waas, Optical Eng., Vol. 46, p. 051005, (2007) 